\title{
Mathematical Education of a Modern Engineer: Problems of Integration
}

\author{
Alexander Novikov, Nikolay Chekalkin, Nadezhda Sokolaeva, Dmitry Miskov, Ekaterina \\ Dzhevello, Evgeniy Larionov, Vyacheslav Muraviev, Maxim Nazarenko
}

\begin{abstract}
The article discusses the methods of teaching mathematics at a technical University, focuses on the means of integration of mathematical and engineering training of students, provides recommendations for the preparation and use of educational and practical tasks that implement the goals of integration in the educational process of the University.

Index Terms: mathematics teaching, engineering education, knowledge integration
\end{abstract}

\section{INTRODUCTION}

Mathematics, at least since the time when engineering education has acquired certain forms and stood out in an independent industry, is considered, of course, useful for the training of future engineers. Moreover, for engineering, it is recognized as a fundamental scientific discipline, and the selection of students, for more than two hundred years, is done largely on their mathematical abilities shown in the entrance exams [1-9]. Contradictions in the relations to mathematics begin in questions of the maintenance of mathematical preparation of the engineer [10-19] and even more in methods of teaching of mathematical courses.

\section{RESEARCH METHOD}

In the history of technical education compete two theoretical and methodological approaches to the didactics of mathematical training engineer. The first one is based on the fact that mathematics has its own internal structure and its own logic, which is absolutely necessary for its understanding, assimilation and ability to use it correctly in applications. There is no separate applied mathematics. It is United and therefore the teaching of mathematics to engineers should not differ significantly from the University course. The second approach States that the goals of studying mathematics and scientific interests of the engineer are different than that of a professional mathematician. Therefore, in engineering education mathematics is something special, "engineering mathematics". It should also be taught in a different way, taking into account the needs of the specialty and the specifics of engineering thinking. The necessary environment of engineering education is created, in which theoretical knowledge and practical skills are taught, assimilated and applied in

Revised Manuscript Received on July 09, 2019

Alexander Novikov, Nikolay Chekalkin, Nadezhda Sokolaeva, Dmitry

Miskov, Ekaterina Dzhevello, Evgeniy Larionov' Vyacheslav Muraviev, Maxim Nazarenko practice, the integration of curricula supporting the system of training is achieved; it is prescribed to establish interdisciplinary relations; to maintain the integration of academic disciplines in the educational process as a duty of the teaching staff of the University. Achievement of the set goals is methodically provided by educational and practical tasks, in which the student develops the ability to apply theoretical knowledge in engineering practice. Considering the content of standards and curricula in terms of methodology, we can conclude that in this case we are dealing with a project-oriented educational technology aimed at the integration of theoretical and practical training of students of technical universities. The use of the project method in national engineering education is not new.

\section{RESULTS AND ANALYSIS}

In the $20 \mathrm{~s}, 30 \mathrm{~s}$ of the last century, this methodological direction was considered promising and was intensively introduced, including in the mathematical training of the future engineer. Then the method of projects did not live up to its expectations. Its application in educational practice usually led to a decrease in the quality of mathematical knowledge and this caused complaints from specialized departments. The reason for the failure of the first experience of the method of projects from the standpoint of modern pedagogical science is explained so that the idea was at first glance correct: to bring learning closer to the process of cognition, to make learning more direct, but did not take into account only one fact, namely that the more elements of immediacy (research, experiment, problematics) there is a desire to make learning, the more measures of collateral should be taken simultaneously. The experience of history teaches that the success of an educational project is largely determined at the level of its methodological developments. This means that the practical implementation of the resource base set by standards and programs, in the case of specific academic disciplines should be supported by the necessary means of methodological support. This refers to special means of methodological support, created taking into account the philosophy and conceptual guidelines, focused on their use in the educational process. The method of teaching mathematics in engineering universities was created and improved for several centuries, so the expected methodological support can not be created overnight. From the position of a teacher of mathematics at a technical University, it can be judged

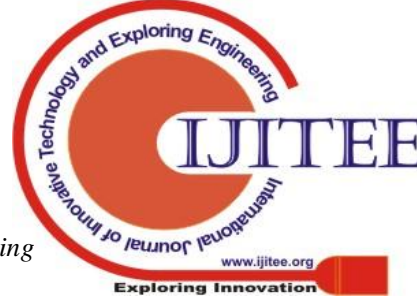


that in the reform of engineering education at the level of a particular discipline, the greatest need exists for explanatory examples that more fully convey the meaning of the plan. Because the teacher in this is no longer a question - "What to do?", and the question- " What means I can achieve this?". Elements of the new philosophy of education, standards, curricula are becoming increasingly common in engineering education in Russia. Mathematics in a technical University should be integrated into the system of engineering education As one of the methodological means of integration, it is proposed to include in the curriculum individual educational and practical tasks (projects) with interdisciplinary content. Since the subject system of education is maintained, and for the teacher of mathematics the highest priority is still the mathematical side of the question, then there is a problem in the methodology of such tasks. The case is quite new and even more difficult that its execution requires knowledge of not only mathematical but also engineering Sciences.

There are six important aspects that reflect the role of mathematics in the training of the future engineer.

1. Establishment of intersubject connections - service of the next disciplines (physics, chemistry, mechanics) that gives them the formalized language for the description of various processes and the phenomena. Thanks to mathematical methods, mechanics, astronomy, physics and other Sciences penetrate into the essence of the laws of nature and foresee what remains beyond the limits of knowledge. Mathematics, contributing to the manifestation of new knowledge about nature, society, finds in related Sciences real incentives for its development. Interdisciplinary connections in teaching mathematics perform educational, developmental, methodological and educational functions. Professional engineering disciplines are a kind of customers of the knowledge and skills that can be formed through mathematics and will be in demand in the future.

2. The practical orientation of the use of mathematical knowledge is the most important component of engineering activity.

Graduates of a technical University must be able to clearly formulate a particular technical problem, build mathematical models, choose the appropriate mathematical method and algorithm for solving the problem, use numerical methods for solving problems, produce and choose practical recommendations based on mathematical analysis.

3. The historical aspect is the transfer to the present generation of previous experience, illustrating and confirming the effectiveness of the use of mathematics in solving various specific problems of any scale - from global to the simplest. It is not by chance that many technical universities, in particular the Russian technological University, have courses in the history of mathematics, where students get acquainted not only with the life and scientific achievements of the great mathematicians, but also study the processes of formation and development of various mathematical disciplines.

4. Ethical aspect-mathematics (as well as literature and art) can be used to solve many vital issues related to self-analysis, analysis and rationalization of their lives and attitudes, culture of work and thinking, the relationship with society and awareness of their place in it; here we can talk about the social significance of mathematical knowledge, which requires the development of the learner's worldview and understanding of the role of mathematics in the formation of the scientific picture of the world, allowing to adequately reflect reality.

5. Aesthetic aspect-the development and formation of associative, creative mathematical thinking, not only to know and understand the studied issues, theorems, methods, but also to feel deeply, feeling their beauty and efficiency, greatness and universality, the limits of applicability and connection with other aspects of human knowledge: music, language, philosophy, physics, literature; the main features of creative mathematical thinking are laconism, the desire to always find the shortest, leading to the goal of a logical path. Creative approach to solving mathematical and technical problems is manifested in the ability to modify a given situation to create conditions for the applicability of a method, or in the ability to design new ones on the basis of this problem, to control and explore the result of the solution.

6. Training aspect-mathematics is an ideal tool for brain training. Mathematics helps to achieve a high level of mental concentration, stability of attention, the ability for a long time to engage in a certain activity, to pay attention to several objects at the same time.

All this leaves an imprint on the specifics of the course of mathematics in a technical University.

Mathematics for an engineer is a tool and a tool. Let us consider some features of the mathematics course for future engineers.

1. The specific weight of the topic related to the graphical representation of the material in the preparation of mathematics and engineering is very significant. Theory for and the other one, it fits in three lectures (with examples), however, if mathematics is one of the important subjects for engineer, it is the most important. In narrow terms, this topic is an ideal battery of all the material of the first semester, in its study of mathematics and engineers are in an equal position. As a result of studying of schedules at students the basic engineering qualities will develop, they will learn to estimate a situation. All engineering mathematics rests on two words - substitute (to bring up the numbers) and evaluate. Learn how to operate these concepts can be engaged in graphical constructions. We note one more important fact, which is very typical for engineering practice. Often a complete study of the function is impossible (due to lack of time or the nature of the situation) or not necessary, but at least some basic features of the function must be seen. In a simplified version, the question is to describe (characterize) the behavior of the function in the domain of definition. Of course, without the use of derivatives, some parts sometimes fall out. But speed justifies everything, and the time factor is often decisive in engineering work. Therefore, it is extremely useful to build charts orally, without calculations, differentiation. Such a facilitated-accelerated 
training will have a beneficial effect in the future, in the study of certain (especially improper) integrals and series. According to the unwritten law of engineering, it is necessary to see at least in General the main features of any function. Unfortunately, the misunderstanding of this simple situation is sinned by many teachers of mathematics, including the authors of the most popular textbooks and problem books the study of the function for plotting is divided into parts: in the problems of one paragraph it is necessary to look only for the intervals of increase or decrease, in others-only asymptotes, etc. This approach is simply harmful.

2. Excessive formalization of submission of material to Junior students. An example of how a good thing becomes not just useless, but also harmful are quantifiers. Students download answers from the Internet, completely without understanding the meaning, and can not Express in words what they mean. Many textbooks of mathematics for students of technical universities sin overload quantifiers. Most students do not perceive text where mathematical symbols are more than words. If there was such a dilemma-the quantifier or the Russian language? - the choice is clear: language is more important.

3. Requirements for knowledge of the material are different for mathematicians and engineers: for the mathematician the most important thing is the breadth of the vocabulary, and for the engineer - the ability to talk. Unfortunately, in language teaching, despite the vast practical experience, there are still no clear criteria for the ratio between the number of words and the compilation of meaningful sentences. Each teacher solves this important task individually, intuitively and automatically. But sooner or later comes the stage of knowledge control, where automatic solutions are impossible. The transition to the written form of examinations greatly complicates the situation, as in the tasks there are different questions-and those on which the student has the right to think, and those to which he is obliged to answer on the move. Here there is a gap with engineering practice: the engineer is often faced with tasks that need to be solved today, as tomorrow will be late. All classes, and especially exams, tests, tests, should be a prototype of engineering work, and the usual game of questions and answers to engineering everyday life is a little similar.

4. Each time has its own laws. Current students are children of the exam and the Internet. The harmfulness of the exam is not a secret for anyone. With mathematics on the Internet can be found partly directly by opening the relevant sites, partly indirectly — on the examination sheets of students (it is clear that they themselves wrote their theory could not come up). Often in the works of students mathematical theory is presented with gross errors and questionable proofs of theorems. For example, a whole page stretches the proof of the theorem, for which the engineer only one line of calculations and several lines of explanation. Or theorems, which are always recommended to write in the standard form with the turnover " If..., then...", suddenly take the form: "Let... . Then.»... But the conditions of the theorem and assumption are two different things, mixing them is clearly beyond the scope of stylistic sloppiness. In this regard, it is necessary to create educational websites, electronic libraries containing publications that have passed a strict review in technical universities. Particular attention should be paid to the creation of electronic lecture notes, electronic textbooks with interactive elements, which contain training elements, questions for self-control, tips. Electronic educational materials should differ significantly from digitized educational and teaching AIDS and contain in addition to the main text of various additions, hyperlinks to educational materials from the electronic library, pop - up Windows with explanations, typical tasks and interactive examples of their solutions, photo and video materials, animation.

5. The whole centuries - old history of scientific knowledge is the relationship of two different methodological approaches: theory and experiment. Both approaches exist on an equal footing, interact, cooperate, and mutually enrich and complement each other. These theories test the experiment data of experiment - theory. There are two characteristic nuances of the relationship between theory and experiment. First, it is becoming more and more active and dynamic: there are almost no natural experiments left, the experiment is gaining a greater initial theoretical basis, without which the experiment is difficult to organize and it is impossible to understand the meaning of its results. Second, the coincidence or the discrepancy between data obtained theoretically and experimentally, it is necessary to evaluate all the more subtly dialectical. Although the best coincidence only indirectly confirms the truth of the result, it gives researchers a reason to believe in this result, to fully recognize it. However, the discrepancy between theoretical and experimental data is most useful for science, as it fixes the underdevelopment of scientific views and thus gives impetus to the development and change of the theory, generates new hypotheses and concepts. The eternal question: what is the lack of coincidence-in theory, experiment, or both of them? Since (in terms of numbers) the theory is pure and the experiment is inaccurate, the requirements for interpretation and correct processing of experimental data are becoming increasingly high. The history of the development of science shows that the role of calculations in theory and in experiment is increasing, calculations are becoming more cumbersome and thin. The emergence of computers and their introduction into scientific use have made a real revolution not only in computing and mathematics, but also in science as a whole. A giant leap in the speed of counting led to the emergence of a new method of studying nature - the possibility of a numerical experiment. We can safely say that now, in addition to theoretical and experimental methods, there is a method of numerical experiment. In a methodological sense, all three approaches are equal, depending on the task, the field of science, one of them is more successful, economical, important, necessary, acceptable. Computational mathematics is now a semi-experimental science.

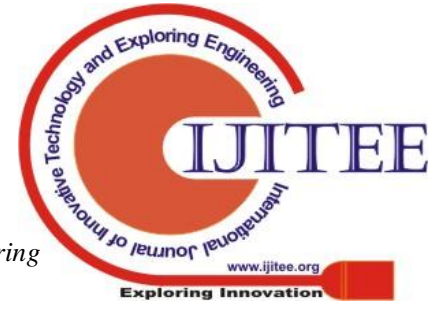


In any case, it cannot be considered just a branch of mathematics. However, it should be taken into account that computational mathematics relies on modern analysis and algebra, using the deepest and most abstract mathematical theorems. Therefore, courses of computational mathematics, numerical methods play a particularly important role in the training of engineers.

6. Improving the level of knowledge of students contributes to the introduction of new teaching methods in the educational process, including the use of modern interactive computer systems, mathematical packages. The use of mathematical packages significantly activates the development of mathematical concepts, theorems and methods of solving problems, as well as contributes to the further implementation of engineering and scientific calculations using mathematical packages. In conjunction with the modular rating system of the educational process, the use of mathematical packages contributes to the development of motivational incentives for students. However, as experience shows, if you force the process of widespread use of mathematical packages in the educational process, you can come to the training senseless pressing the buttons, after which the integral will be associated with the student only with the hook. We can say that the growth of requirements for engineering calculations leads to an increase in requirements for the level of mathematical knowledge of future engineers. The knowledge obtained by the engineer should reflect the specifics of professional activity; the ability to use mathematical and numerical methods, MP, modern interactive computer systems in engineering calculations. The future engineer should realize the importance of mathematics as an integral part of human culture, understand that without knowledge of mathematics he will not be able to realize the practical tasks facing him.

\section{CONCLUSION}

Integrating educational and practical task in mathematics should be in the form of a technical project, and combine in its formulation of the engineering problem with mathematical methods of research. Of course, with a mandatory emphasis on emerging in the study of the object of technology mathematical problems. The formulation of such a problem causes problems for the student, accustomed to solving only typical problems at the lessons of mathematics, is accompanied by significant difficulties. Therefore, for didactic purposes, it is recommended to divide the task into two parts: in the first (propaedeutic) - the teacher directly leads the student, participating in the formulation of mathematical problems and the choice of the method of solution, makes comments and step by step controls the progress of the task; in the second (creative) - the student independently solves a similar problem, having the opportunity to use the methodological techniques of the first part; or he is invited to improve the design of the first part, which in this case functionally sets the required mathematical apparatus and you step as a prototype of the project.

\section{Conflict of Interest}

The authors declare no conflict of interest.

\section{REFERENCES}

1. N.B. Golovanova et al, "Implementation Of The Practically-Oriented Approach In The Specialists Training On Radio Engineering And Communication Systems" Rossiyskiy tekhnologicheskiy zhurnal (Russian Technological Journal). 2018. 6(1): 73-87. (in Russ.)

2. S.V. Kruchinin et al, "Main Self-Educational Platforms in Russia and Skills that Can Be Develop Via Them" Proceedings of the 2018 International Conference "Quality Management, Transport and Information Security, Information Technologies", IT and QM and IS, 2018, 8525032, p. 669-671. DOI: 10.1109/ITMQIS.2018.8525032

3. E.V. Bagrova et al, Additional Education in Russia. Ways of Development" Proceedings of the 2018 International Conference "Quality Management, Transport and Information Security, Information Technologies", IT and QM and IS, 2018, 8524999, p. 561-564. DOI: 10.1109/ITMQIS.2018.8524999

4. S.V. Kruchinin et al, "Simultaneous Development of E-Learning in Russia and Information Society" Proceedings of the 2018 International Conference "Quality Management, Transport and Information Security, Information Technologies", IT and QM and IS, 2018, 8524994, p 666-668. DOI: 10.1109/ITMQIS.2018.8524994

5. A.A. Filippov et al, "Algorithm of End-to-End Integrated Quality Management" Proceedings of the 2018 International Conference "Quality Management, Transport and Information Security, Information Technologies", IT and QM and IS, 2018, 8525039, p. 44-46. DOI: 10.1109/ITMQIS.2018.8525039

6. M.S. Kostin et al, "Counterreengineering of electronic devices" Rossiyskiy tekhnologicheskiy zhurnal (Russian Technological Journal). 2019; 7(1) 57-79. (in Russ.). DOI: 10.32362/2500-316X-2019-7-1-57-79

7. V.V. Muraviev et al, "Company Life Cycle and Quality Standardization" Proceedings of the 2018 International Conference "Quality Management, Transport and Information Security, Information Technologies", IT and QM and IS, 2018, 8525064, p. 59-62. DOI 10.1109/ITMQIS.2018.8525064

8. S.V. Kruchinin et al, "Key Ways of Information Technologies for Self-Education Development. Pros and Cons" Proceedings of the 2018 International Conference "Quality Management, Transport and Information Security, Information Technologies", IT and QM and IS, 2018, 8544901, p. 663-665. DOI: 10.1109/ITMQIS.2018.8524901

9. M.A. Nazarenko et al, "Model end-to-end integrated quality management" International Journal of Engineering and Technology (UAE). 2018. Volume 7, Issue 4.36. P. 60-63.

10. E.V. Bagrova et al, "Usage of Information Technologies in Self-Education in Russia" Proceedings of the 2018 International Conference "Quality Management, Transport and Information Security, Information Technologies", IT and QM and IS, 2018, 8524940, p. 565-567. DOI: 10.1109/ITMQIS.2018.8524940

11. M.A. Nazarenko, "Mobile Operating Systems and Integrated Apps Quality Management System" Proceedings of the 2018 International Conference "Quality Management, Transport and Information Security, Information Technologies", IT and QM and IS, 2018, 8525022, p. 63-65. DOI: 10.1109/ITMQIS.2018.8525022

12. E.S. Djevello et al, "Specifics of Management Quality Implementation to Information Systems and Products" Proceedings of the 2018 Internationa Conference "Quality Management, Transport and Information Security, Information Technologies", IT and QM and IS, 2018, 8524995, p. 40-43. DOI: 10.1109/ITMQIS.2018.8524995

13. E.V. Bagrova et al, "Quantity Measures of Quality in Higher Education in Russia. How Information Technologies Can Help?" Proceedings of the 2018 International Conference "Quality Management, Transport and Information Security, Information Technologies", IT and QM and IS, 2018, 8525044, p. 557-560. DOI: 10.1109/ITMQIS.2018.8525044

14. M.A. Nazarenko, T.V. Khronusova "Big Data in Modern Higher Education. Benefits and Criticism" Proceedings of the 2017 International Conference "Quality Management, Transport and Information Security, Information Technologies", IT and QM and IS, 2017, 8084914, p 676-679. DOI: 10.1109/ITMQIS.2017.8085914

15. A.I. Gorobets et al, "Technical and economical analysis of efficiency of electronic means' quality management" International Journal of Engineering and Technology (UAE). 2018. Volume 7, Issue 4.20. P. 629-630. DOI: 10.14419/ijet.v7i2.8.10548

16. M.A. Nazarenko, "Some properties of rational approximations of degree $(\mathrm{k}, 1)$ in the Hardy space H2(D)" 
Mathematical Notes. 1998. 64(1-2). P. 213-219.

17. M.A. Nazarenko et al, "Main directions of higher education regionalization process as an integral part of social partnership in society (The problems of inclusive education)" International Journal of Engineering and Technology (UAE). 2018. Volume 7, Issue 4.36. P. 1135-1138. DOI: 10.14419/ijet.v7i4.36.25052

18. Y.Y. Cheremukhina et al, "The process of working with claims in the quality management system of an enterprise of the radio-electronic industry" International Journal of Engineering and Technology (UAE). 2018. Volume 7, Issue 4.36. P. 67-68. DOI: 10.14419/ijet.v7i4.36.23313

19. M.A. Nazarenko et al, "Antivirus software and industrial cyber security system certification in Russia" Rossiyskiy tekhnologicheskiy zhurnal (Russian Technological Journal). 2019; 7(1): 48-56. (in Russ.). DOI: $10.32362 / 2500-316 \mathrm{X}-2019-7-1-48-56$ 